\title{
Tensile and fatigue assessments of brazed stainless steel joints using digital image correlation
}

\author{
Anke Schmiedt ${ }^{1, *}$, Simon Jaquet ${ }^{1}$, Matthias Manka ${ }^{2}$, Wolfgang Tillmann ${ }^{2}$, and Frank Walther ${ }^{1}$ \\ ${ }^{1}$ TU Dortmund University, Department of Materials Test Engineering (WPT), Baroper Str. 303, D-44227 Dortmund, Germany \\ ${ }^{2} \mathrm{TU}$ Dortmund University, Institute of Materials Engineering (LWT), Leonhard-Euler-Str. 2, D-44227 Dortmund, Germany
}

\begin{abstract}
For mechanical tests of brazed stainless steel joints, the local deformation behaviour within the small area of the brazing seam is a major concern, because local strains cannot be detected with standard mechanical extensometers. The current study allows a fundamental comprehension of the gauge length influence on the strain measurements of brazed joints with smooth and notch-containing surfaces, under quasistatic and cyclic loadings. Therefore, the optical measurement technique of digital image correlation (DIC) is used within tensile and fatigue tests of brazed AISI 304L/BAu-4 joints in an as-received and pre-corroded condition. A triggered image acquisition of the DIC system is successfully applied to evaluate the local ratcheting fatigue behaviour in the area of the brazing seam at a frequency of $10 \mathrm{~Hz}$. The gauge length influence, analysed in the range of 0.5 to $12.5 \mathrm{~mm}$, is more pronounced with increasing tensile and fatigue stresses and is significantly enhanced for notch-containing surfaces. Instrumented load increase tests with strain, electrical, magnetic and temperature measuring techniques have proven to be appropriate to estimate fatigue properties of the brazed joints with a deviation of $4 \%$. Fatigue and corrosion fatigue damage mechanisms are evaluated by using scanning electron microscopy with secondary and back-scattered electron detectors.
\end{abstract}

\section{Introduction}

Brazing technologies are well established for an efficient and economic production of joined structures for numerous reliable applications, in particular for the automotive industry, chemical engineering and power generation. The potential and benefits of the joining technology, considering e.g. thermal stresses, material combinations, complex structures and process times, have already been extensively described [1]. Until now, several studies have been realised in order to investigate the mechanical properties as well as the damage and fracture behaviour of brazed joints under quasi-static and cyclic loadings [2]. For the evaluation of the strain measurements, deviating deformation behaviours of the brazing seam from the base material have to be considered. It is known, that local strain concentrations cannot be detected with standard extensometers and a significant influence of the gauge length is present.

The optical measurement technique of digital image correlation (DIC) was developed for a 3-dimensional, non-contact and full-field image analysis in order to generate deformation and strain maps across an entire material surface. Most of the investigations utilizing the DIC technique were performed with structures under static loads, aiming at a visualisation of the strain distribution [3]. Based on the local strain and stress concentrations, potential failure locations can be identified. An application of the optical technique, using standard digital cameras, for mechanical fatigue tests leads to a particular challenge, as common loading frequencies of $10 \mathrm{~Hz}$ already require a complex triggered image acquisition. Up to now, fatigue assessments using the DIC technique for the evaluation of the continuous cyclic deformations behaviours are relatively rare [3].

The high potential of the DIC technique to determine fatigue-induced damage evolutions in brazed steel joints was already presented [1]. Within this current study, the deformation and damage behaviour of AISI 304L/BAu-4 brazed stainless steel joints is analysed under quasi-static and fatigue loadings using a DIC system as well as standard mechanical extensometers for validation. A triggered image acquisition enables an evaluation of the continuous cyclic creep properties under fatigue loading with a frequency of $10 \mathrm{~Hz}$. In addition to strain maps, various virtual strain gauges are placed post-test in the area of the brazing seam in order to demonstrate the influence of the gauge length of standard extensometers in mechanical tests of brazed specimens. For the fatigue assessment, time-efficient, instrumented stepwise load increase tests (LIT) are performed with the aim to determine the local strain distributions within the total stress range, leading to elastic and plastic material deformations. The applicability of the DIC technique for non-smooth surfaces is evaluated in tensile and fatigue tests of pre-corroded brazed specimens. For comparison, homogeneous base material specimens are analysed.

\footnotetext{
* Corresponding author: anke.schmiedt $@$ tu-dortmund.de
} 


\section{Materials and experimental procedure}

\subsection{Materials}

The austenitic stainless steel X2CrNi18-9 (AISI 304L; 1.4307 ), acc. to specification DIN EN 10088-3, is widely used in industry and was utilised as base material for the investigated brazed butt joints. The chemical composition, acc. to the test certificate, is listed in Tab. 1. Plastic deformations of the metastable austenite can lead to a phase transformation to ferromagnetic martensite. This fact was extensively investigated in terms of cyclic loadings for homogenous alloys in earlier studies [4-6]. The deformation-induced martensitic transformation, which is sensitive to the test temperature, strongly affects the cyclic deformation behaviour and the lifetime of metastable austenites. Hence, the self-heating of the material caused by cyclic loadings has to be taken into consideration. This effect was evaluated in anisothermal and isothermal fatigue tests at room temperature with and without cooling [5-7]. As a result of high temperatures due to self-heating, the martensitic transformation can be inhibited. In contrast, martensitic transformations in isothermal tests lead to a secondary cyclic hardening, which increases the lifetime [7].

A commercially available gold-based brazing alloy BAu-4 (Au 827), with the chemical composition Au82-Ni18, was applied as a $50 \mu \mathrm{m}$ amorphous brazing foil between the base material prior to the brazing. The brazing process was carried out in a vacuum furnace with a vacuum of $10^{-5}$ to $10^{-6} \mathrm{mbar}$, using a brazing temperature of $1050{ }^{\circ} \mathrm{C}$ in conjunction with a short dwell time of $2 \mathrm{~min}$, a heat treatment for $2 \mathrm{hrs}$ at $950{ }^{\circ} \mathrm{C}$ and a subsequent free cooling down to room temperature. The microstructure of the brazed AISI 304L/BAu-4 joints in the as-received condition is shown in Fig. 1a.

Specimens for tensile and fatigue tests were produced from brazed cylindrical bars with the same specimen geometry, Fig. 2. The rejuvenated area was polished in order to obtain a surface quality of $\mathrm{R}_{\mathrm{z}}=0.9 \mu \mathrm{m}$ and to ensure the consistency and reproducibility of the test results. In contrast to standardised tensile test specimens, the applied geometry shows a shorter gauge length of $12.5 \mathrm{~mm}$. As a result, an increase of the elongation values during tensile tests is expected, if necking occurs. For the base material specimens, the AISI 304L cold drawn bars were heat-treated acc. to the temperature-time curve of the brazing process and manufactured as described in [8]. Before testing, a white speckle pattern was generated with an airbrush system on the black coated specimens for the DIC analysis.

Tab. 1. Chemical composition of AISI 304L base material acc. to test certification.

\begin{tabular}{|c|c|c|c|c|c|c|c|c|}
\hline \multicolumn{10}{|c|}{ Alloying elements, wt.-\% } \\
\hline $\mathbf{C}$ & $\mathbf{C r}$ & $\mathbf{N i}$ & $\mathbf{M n}$ & $\mathbf{S i}$ & $\mathbf{P}$ & $\mathbf{S}$ & $\mathbf{N}$ & $\mathbf{F e}$ \\
\hline 0.018 & 18.23 & 8.06 & 1.05 & 0.42 & 0.035 & 0.027 & 0.074 & bal. \\
\hline
\end{tabular}
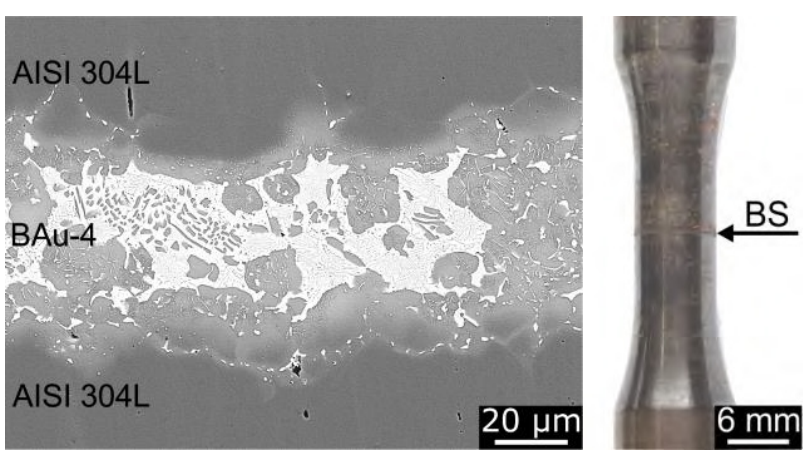

a)

b)

Fig. 1. a) Microstructure of the brazing seam (BS) in the as-received condition; b) Specimen after pre-corrosion.

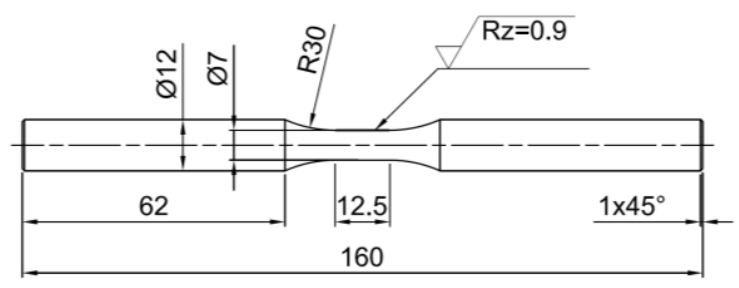

Fig. 2. Specimen geometry for tensile, fatigue and corrosion fatigue investigations (all dimensions in $\mathrm{mm}$ ).

The prepared AISI 304L/BAu-4 and AISI 304L specimens were pre-corroded acc. to the VDA test 230-214 [9], which is established for exhaust gas-carrying components. Therefore, the DIC technique was evaluated for surfaces that are not smooth but notch-containing. The alternating corrosion exposure consists of daily periods of semi-immersion, drying and a steam phase in combination with a weekly heat ageing, conducted at $600{ }^{\circ} \mathrm{C}$ for the base material and at $400{ }^{\circ} \mathrm{C}$ for the brazed AISI 304L/BAu-4 joints. After 6 weeks of pre-corrosion, depositions of corrosion products can be detected on the specimen's surfaces. In the area of the brazing seam, the corrosive attack leads to a local circumferential metal dissolution, especially at the interface to the base material, Fig. $1 \mathrm{~b}$.

\subsection{Experimental details}

The deformation behaviour of AISI 304L/BAu-4 brazed stainless steel joints was analysed under quasi-static and cyclic loadings using a DIC system and standard mechanical extensometers. Tensile tests were performed with the base material and the brazed specimens prior to and after 6 weeks of pre-corrosion in order to evaluate the applicability of the DIC system for homogeneous and joint geometries as well as for smooth and non-smooth surfaces. For fatigue tests, brazed specimens of both conditions were used. Two specimens were tested for each material, condition and mechanical loading.

Quasi-static tensile tests were performed at a universal testing system (Shimadzu, $F=100 \mathrm{kN}$ ) with and without using an extensometer with a gauge length of $10 \mathrm{~mm}$ (GL10). Applying the extensometer allows to compare the DIC and standard strain measurements, while the complete gauge length of $10 \mathrm{~mm}$ can only be evaluated optically if the mounting springs of the 
extensometer do not obscure the view on the specimen's surface. In accordance with ISO 6892, the testing speed can be increased after the yield strength is determined. Up to the total strain $\varepsilon_{\mathrm{t}}$ in the range of 8 to $14 \%$, tests with an extensometer were constantly controlled with a strain rate of $\dot{\varepsilon}=0.00025 \mathrm{~s}^{-1}$. For tests without the extensometer, the crosshead separation rate was constantly controlled by $\mathrm{v}_{\mathrm{c}}=0.8 \mathrm{~mm} / \mathrm{min}$, which correlates with an estimated strain rate over the parallel length $\dot{\mathrm{e}}_{\mathrm{Lc}}=0.00025 \mathrm{~s}^{-1}$. Subsequently, $\mathrm{v}_{\mathrm{c}}=4.8 \mathrm{~mm} / \mathrm{min}$ was controlled until the failure of the specimen.

Fatigue tests were carried out using a servo hydraulic testing system (Instron, $\mathrm{F}= \pm 100 \mathrm{kN}$ ) and an extensometer with a gauge length of $12.5 \mathrm{~mm}$ (GL12.5), featured with a considerable measuring range of $\pm 5 \mathrm{~mm}$ and allowing strain measurements of up to $\pm 40 \%$. The tests were performed by applying a stress ratio of $\mathrm{R}=0.1$ and a frequency of $\mathrm{f}=10 \mathrm{~Hz}$, using sinusoidal load time functions. In addition to constant amplitude tests (CAT) with a limited number of cycles $\mathrm{N}=2 \cdot 10^{6}$, a time- and source-efficient procedure, based on stepwise load increase tests, was applied, which is already established for a wide range of material classes and joints [10, 11]. With a constant stress ratio, the maximum stress was increased stepwise by $\Delta \sigma_{\max }=10 \mathrm{MPa}$ each $\Delta \mathrm{N}=10^{4}$ cycles, starting at a stress level of $50 \mathrm{MPa}$ at which the material and joints are expected to be damage-free up to the level of specimen`s failure.

In addition to the DIC system and the extensometer, the material response to the cyclic loading was continuously evaluated with electrical, magnetic and temperature measuring techniques. Therefore, the experimental setup for fatigue tests includes a crack growth monitor (Matelect) for alternating current (AC) potential drop measurements, a feritscope (Helmut Fischer) as well as high resolution thermal imaging cameras (InfraTec; Micro-Epsilon), Fig. 3. An alternating current power supply was fixed to the clamping jaws and the change in the deformation-induced electrical voltage $\Delta \mathrm{U}$, referring to the initial value at the test beginning, was recorded in the gauge length using two welded wires. Furthermore, the feritscope sensor was positioned at the base material close to the brazing seam in order to detect the change in magnetic portion $\Delta \zeta$ close to the surface, which can be correlated with the plasticity-induced phase transformation of the austenitic AISI 304L base material to ferromagnetic martensite.

The thermal imaging camera allows a visualisation of the temperature distribution until failure of the specimens with a temporal and spatial resolution. Differences down to $20 \mathrm{mK}$ can be measured with the camera detector format of $640 \times 512$ pixels. As recommended in [6], three temperatures were evaluated after testing at the middle of the gauge length $\left(T_{1}\right)$ and at the shafts of the specimen $\left(T_{2}\right.$, $\mathrm{T}_{3}$ ) for calculation of the change in temperature $\Delta \mathrm{T}=\mathrm{T}_{1}-0.5 \cdot\left(\mathrm{T}_{2}+\mathrm{T}_{3}\right)$.

In addition to the tactile measurements, strain measurements within tensile and fatigue tests were performed, using 3D-digital image correlation (Limess) in order to determine the strain distribution at the specimen's surface until failure and to identify the local damage mechanisms for the brazed joints and base materials in different VDA pre-corrosion conditions. Using the DIC technique, the deformation state can be visualised with a temporal and spatial resolution. For fatigue investigations, specific modifications of the DIC system allow a frequency-dependent triggered image acquisition to continuously analyse the cyclic deformation behaviour during fatigue testing at a frequency of $\mathrm{f}=10 \mathrm{~Hz}$. Engineering tangential strains $\varepsilon_{\mathrm{xx}}$, computed post-test in tensile direction for line elements (LE) with various lengths, show the influence of the gauge length in mechanical tests of brazed joints. Asymmetric cyclic loadings with mean stresses lead to a directional accumulation of inelastic strains, which are well known as cyclic ratcheting strains. For the evaluation of ratcheting strains, the total mean strain $\varepsilon_{\mathrm{m}, \mathrm{t}}$ and the total maximum strain $\varepsilon_{\text {max, }}$, plotted as functions of the number of load cycles $\mathrm{N}$, can be considered, respectively. In this context, the total maximum strain $\varepsilon_{\text {max, }}$ is determined by $\varepsilon_{\mathrm{max}, \mathrm{t}}=\varepsilon_{\mathrm{m}, \mathrm{t}}+\varepsilon_{\mathrm{a}, \mathrm{t}}$ with the total strain amplitude $\varepsilon_{\mathrm{a}, \mathrm{t}}$. Within the current study, the ratcheting fatigue and cyclic deformation behaviour of brazed AISI $304 \mathrm{~L} / \mathrm{BAu}-4$ joints were investigated based on $\varepsilon_{\text {max,t }}$, computed for various gauge lengths. Furthermore, the DIC results were compared with strain measurements of standard extensometers.

Within LIT, the transition from almost steady to significantly deviating material reactions at $\sigma_{\text {max,e (LIT) }}$ as well as the failure maximum stress $\sigma_{\max , \mathrm{f}(\mathrm{LIT})}$ have proven to be characteristic for the fatigue behaviour of various materials and joints $[10,11]$. For metastable austenitic materials, a particular evaluation method of LIT was recommended in the literature [6]. It was shown that the high cycle fatigue strength corresponds well with the stress level, at which the cyclic hardening, due to the deformation-induced formation of martensite, leads for the first time to a temperature decrease [6]. Within the current study, the applicability of the evaluation method for brazed AISI 304L/BAu-4 joints in the as-received condition were examined based on load increase and constant amplitude tests. Further, LIT enable the assessment of the DIC technique for the complete stress range, leading to elastic as well as plastic deformations of the brazed joints. After testing, the fracture surfaces were analysed using scanning electron microscopy (SEM), with a secondary electron (SE) and a back-scattered electron (BSE) detector.

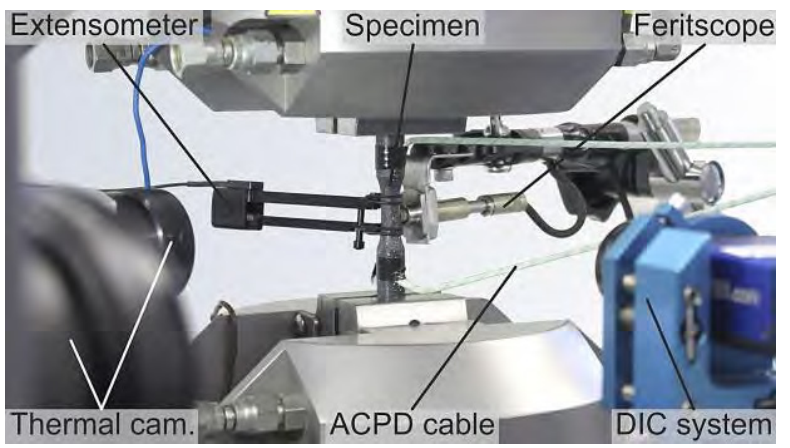

Fig. 3. Experimental setup for fatigue investigations in air. 


\section{Results and discussions}

\subsection{Tensile behaviour}

Quasi-static tensile tests were performed with specimens of the AISI $304 \mathrm{~L}$ base material (BM) and the brazed AISI 304L/BAu-4 joints (BJ) in the as-received (0w.) and 6 weeks pre-corroded (6w.) conditions to assess the DIC technique for homogeneous and joint geometries. According to the deformation state of a base material specimen, stresses below $\sigma_{U T S}$ lead to homogeneous deformations within the rejuvenated area, Fig. 4. Close to failure at a nominal stress $\sigma_{\mathrm{N}}=400 \mathrm{MPa}$, a local higher degree of deformation is detected in the necking area.

The DIC software is used to compute engineering tangential strains in tensile direction $\varepsilon_{\mathrm{xx}}$ for a $10 \mathrm{~mm}$ line element (LE10) in the centre of the specimen with the aim to evaluate the stress-strain curves [8]. As expected, the phenomenon of necking occurred within a major part of the measuring length. Thus, in comparison to standardised specimen geometries, an increase of strain values has to be considered. Comparable stress-strain curves are determined for the AISI 304L prior to and after 6 weeks of pre-corrosion, Fig. 5. Consequently, the long-time corrosive attack does not influence the quasi-static tensile behaviour of the base material, concerning strength as well as deformation properties. Two specimens were tested for both corrosion conditions and average values of the $0.2 \%$ yield strengths and ultimate tensile strengths with small standard deviations were determined with $\sigma_{\mathrm{YS}}=232 \pm 4 \mathrm{MPa}$ and $\sigma_{\mathrm{UTS}}=618 \pm 3 \mathrm{MPa}$. [8]

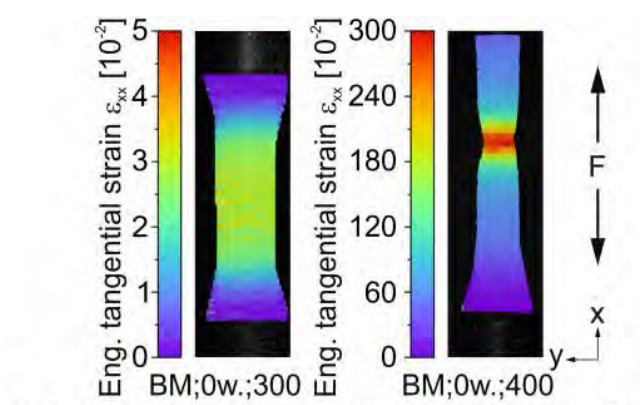

Material; Pre-corrosion; Nominal stress $\sigma_{\mathrm{N}}[\mathrm{MPa}]$

Fig. 4. DIC analysis for a tensile specimen of the base material prior to pre-corrosion at two different stress levels [8].

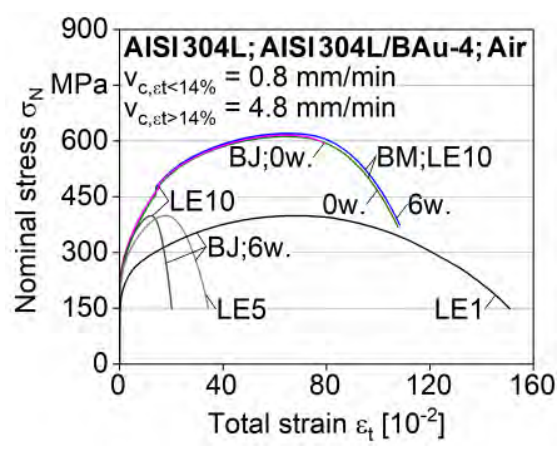

Fig. 5. Quasi-static stress-strain curves of the base material and the brazed joints prior to and after pre-corrosion.
For AISI 304L/BAu-4 brazed joints in the as-received and pre-corroded conditions, the DIC system allows to visualise local strain concentrations in the area of the brazing seam, Fig. 6 . For the smooth surface of the as-received specimen, locally increased strain values can be barely identified for the nominal stress of $300 \mathrm{MPa}$, while a clear distinction between the base material and the brazing seam is possible after the $\sigma_{U}$ is is reached, Fig. 6. In addition to the qualitative evaluation, the engineering tangential strains $\varepsilon_{\mathrm{xx}}$ are computed with the DIC software for line elements with lengths of $0.5,1,3$ and $5 \mathrm{~mm}$ (LE0.5, LE1, LE3, LE5), positioned at the brazing seam with the aim to evaluate the influence of the measurement length on the stress-strain curve. The maximum gauge length within these tests is limited to $5 \mathrm{~mm}$ due to the mounting springs of the extensometer, which was removed at $\varepsilon_{\mathrm{t}}=8 \%$.

In contrast to the plastic deformation range, there is no effect of the gauge length on the elastic modulus, Fig. 7. Comparing the 0.5 and $5 \mathrm{~mm}$ line elements, the difference in the total strain $\Delta \varepsilon_{\mathrm{t}}$ constantly increases with increasing stresses from e.g. $\Delta \varepsilon_{\mathrm{t}}=0.2 \%$ at $300 \mathrm{MPa}$ up to $5.0 \%$ at $\sigma_{\text {UTS }}$, Fig. 7 . The total strains close to failure show the greatest difference of $\Delta \varepsilon_{\mathrm{t}}=11.8 \%$. Consequently, the DIC system is well suited to evaluate local strain concentrations in the area of the AISI 304L/BAu-4 brazing seam for specimens with a smooth surface. The stress-strain curve, evaluated with the DIC system, is validated up to $\varepsilon_{\mathrm{t}}=8 \%$ using an extensometer with a $10 \mathrm{~mm}$ gauge length (GL10), Fig. 7. Thus, mechanically determined strain values are well located within the range of the strain values, computed with the DIC system.

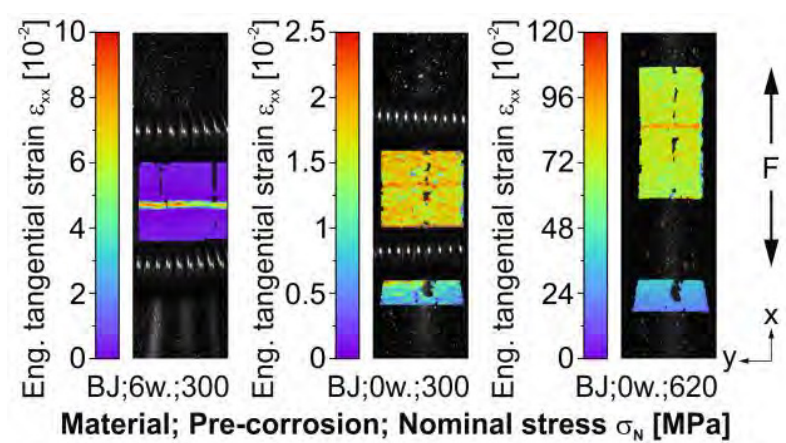

Fig. 6. DIC analysis for brazed tensile specimens prior to and after pre-corrosion.

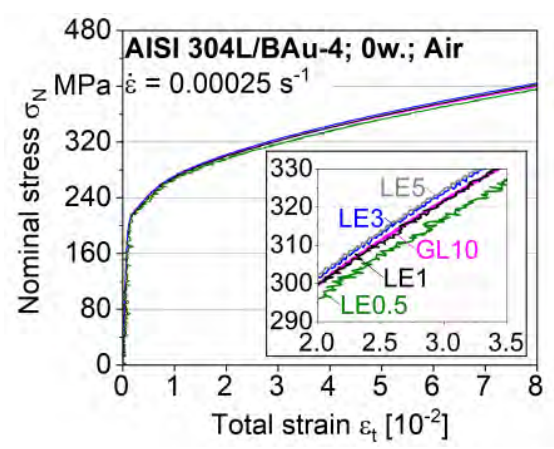

Fig. 7. Quasi-static stress-strain curves of the brazed joints prior to pre-corrosion. 
Additional tensile tests of the brazed joints prior to and after pre-corrosion are performed without the extensometer in order to evaluate the total strains of a $10 \mathrm{~mm}$ line element (LE10) for direct comparison with the results of the base material, Fig. 5. Comparable stress-stain curves are determined for the base material and the brazed joints in the as-received condition and a significant degradation of the yield and ultimate tensile strengths due to the brazing process do not have to be considered. As the neck phenomenon is less distinct for the brazed specimens, the maximum total strain close to failure is reduced from $108 \%$ of the base material to $79 \%$. For the brazed AISI $304 \mathrm{~L} / \mathrm{BAu}-4$ joints, the VDA pre-corrosion for 6 weeks leads to a reduction of the $\sigma_{U T S}$ down to $65 \%\left(\sigma_{U T S}=399 \mathrm{MPa}\right)$ and to a significant decrease of the total strain close to failure down to $\varepsilon_{\mathrm{t}}=20 \%$. Here, the applied nominal tensile stress $\sigma_{\mathrm{N}}$ is calculated based on the gross cross-sections.

The effect of the pre-corrosion on the deformation state of a brazed tensile specimen is presented for the nominal stress of $300 \mathrm{MPa}$, Fig. 5. The local strain increase at the brazing seam is more pronounced due to the corrosive attack. Thus, expected higher local degrees of deformation at notches under tensile stresses, especially due to splitting effects, are confirmed. For line elements with a length of 1 and $5 \mathrm{~mm}$ (LE1, LE5), the difference in the total strain of $\Delta \varepsilon_{\mathrm{t}}=49 \%$ at $\sigma_{\mathrm{UTS}}$ and of $\Delta \varepsilon_{\mathrm{t}}=117 \%$ close to failure were computed, Fig. 5 . Due to the significant opening of the corrosion-dependent notch, an evaluation of the $0.5 \mathrm{~mm}$ line element was not possible. Consequently, a considerable influence of the gauge length on the strain values was determined with the DIC system for the pre-corroded brazed joints.

\subsection{Fatigue behaviour}

The material reactions within LIT of the brazed AISI $304 \mathrm{~L} / \mathrm{BAu}-4$ specimens in the as-received and pre-corroded conditions were recorded by strain, electrical, magnetic, and temperature measuring techniques in order to evaluate the deformation and damage behaviour. The progression of the controlled maximum stress $\sigma_{\max }$ as well as the total strain amplitude $\varepsilon_{\mathrm{a}, \mathrm{t}}$, the total maximum strain $\varepsilon_{\max , t}$, the loss energy density $\mathrm{w}$, the change in $\mathrm{AC}$ voltage $\Delta \mathrm{U}$ and the change in temperature $\Delta \mathrm{T}$ are plotted as functions of the load cycles $\mathrm{N}$ for the as-received brazed joint, Fig. 8. The presented strain measurements were conducted with an extensometer with a gauge length of $12.5 \mathrm{~mm}$ (GL12.5).
Up to a stress level of approx. $\sigma_{\max }=290 \mathrm{MPa}$ at $\mathrm{N}=24 \cdot 10^{4}$ cycles, values of $\varepsilon_{\mathrm{a}, \mathrm{t}}$ and $\varepsilon_{\text {max }, \mathrm{t}}$ remain constant within the stress levels and increase linearly with increasing stress. Afterwards, $\varepsilon_{\mathrm{a}, \mathrm{t}}$ still shows a stepwise increase, while a more continuous growth is determined for the data of the total maximum strain $\varepsilon_{\text {max,t. }}$. For stresses above $290 \mathrm{MPa}$ and up to failure of the specimen at $47.4 \cdot 10^{4}$ cycles $\left(\mathrm{N}_{\mathrm{f}(\mathrm{LIT})}\right)$, both curves show a pronounced progressive increase in combination with a degressive trend within the constant stress levels. At the failure maximum stress $\sigma_{\max , \mathrm{f}(\mathrm{LTT})}=520 \mathrm{MPa}$, a considerable total maximum strain $\varepsilon_{\max , t}$ of approx. $30 \%$ is reached. The determined directional accumulation of plastic strain is a result of an asymmetric cyclic loading with mean stresses and is well known as ratcheting fatigue. The ratcheting strains in form of the total maximum strain $\varepsilon_{\mathrm{max}, \mathrm{t}}$ and the total mean strain $\varepsilon_{\mathrm{m}, \mathrm{t}}$, respectively, are known to be more pronounced for elevated mean stresses and stress amplitudes [11]. The degressive trend of the cyclic deformation curves within the constant stress levels corresponds well with results in the literature on the AISI 304L austenite [12]. Within the first load cycles, the total maximum strain $\varepsilon_{\text {max, }}$ significantly increases and is expected to be a result of the rearrangement of the dislocation structure, until it reaches a more stable configuration [12]. Subsequently, slightly increasing ratcheting rates are observed within the main part of the stress levels.

The measured values concerning the change in $\mathrm{AC}$ voltage $\Delta \mathrm{U}$ remain at a constant level until $24 \cdot 10^{4}$ cycles and finally increase progressively until failure. Degressive curve progressions within the constantly controlled stress levels, as well as the trend throughout the entire fatigue life, correspond well with the total maximum strain $\varepsilon_{\text {max,t. }}$ Thus, the electrical measuring technique is well suitable to characterise the cyclic deformation behaviour of brazed AISI 304L/BAu-4 joints to indicate damage-induced microstructural changes as well as to announce fatigue fractures at $50 \%$ of fatigue life.

The energy dissipation during the cyclic loading of the brazed joints at $\mathrm{f}=10 \mathrm{~Hz}$ leads to a self-heating of the specimen with a maximum temperature in the gauge length $\mathrm{T}_{1}$ of $41.6^{\circ} \mathrm{C}$ and corresponds with a maximum change in temperature $\Delta \mathrm{T}$ of $7.2 \mathrm{~K}$ at failure. A local temperature increase in the area of the brazed joint could not be determined with the high resolution thermal imaging camera, which allows to measure smallest temperature differences of $20 \mathrm{mK}$.

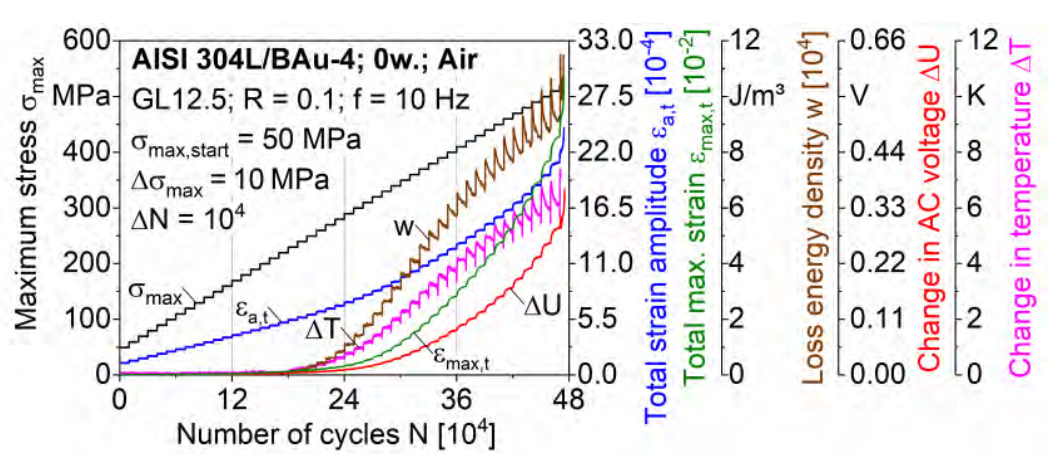

Fig. 8. Load increase test of a brazed specimen in the as-received condition. 
The temperature of the specimen increases linearly until $20 \cdot 10^{4}$ cycles, followed by a progressively increasing trend, with respect to the average values of each stress level until failure. In contrast, a regressively decreasing temperature has to be considered for higher stresses, first detected at $\sigma_{\max }=380 \mathrm{MPa}$. The decrease of the change in temperature is more pronounced with increasing stresses. In detail, the change in temperature $\Delta \mathrm{T}=7.0 \mathrm{~K}$, measured at the beginning of the stress level $\sigma_{\max }=510 \mathrm{MPa}$ at $\mathrm{N}=46.1 \cdot 10^{4}$ cycles, decreases to $\Delta \mathrm{T}=6.1 \mathrm{~K}$ after $10^{4}$ cycles, Fig. 9 .

In order to evaluate the decreasing temperatures as an indicator for the energy dissipation during fatigue loading, the cyclic deformation behaviour of the brazed joints is evaluated by stress-strain hysteresis loops. The areas of the hysteresis loops are calculated with $\mathrm{w}=\oint \sigma \mathrm{d} \varepsilon[4]$ and the progression of the loss energy density $\mathrm{w}$ is plotted as a function of the load cycles $\mathrm{N}$. In good agreement with the change in temperature $\Delta \mathrm{T}$, the loss energy density increases stepwise and linearly up to $20 \cdot 10^{4}$ cycles and, subsequently, progressively increases until failure, when considering the values at the beginning of the stress levels. For higher stresses, the loss energy density regressively decreases and therefore, indicates a cyclic hardening effect. The first decrease is detected at $\sigma_{\max }=350 \mathrm{MPa}$, which is three stress levels below the first detected temperature decrease. In accordance with the temperature, the decrease is more pronounced with increasing stresses with a maximum change of $1.5 \cdot 10^{4} \mathrm{~J} / \mathrm{m}^{3}$ within the last completely tested stress level of $\sigma_{\max }=510 \mathrm{MPa}$. The direct correlation of the temperature and the cyclic deformation behaviour was already explained with the fact that $95 \%$ of the loss energy density dissipates in heat [4].

The cycles hardening of metastable austenitic stainless steels, such as AISI 304L, was extensively investigated in the literature and is known to be mainly a result of a deformation-induced martensite formation $[4-7,12]$. The enhanced strength of the martensitic phase leads to a significant change of the cyclic deformation behaviour and increases the fatigue lifetime [7]. The austenite stability depends on the chemical composition of the austenite, on the temperature as well as on the degree of plastic deformation [12]. Here, a maximum temperature change of $\Delta \mathrm{T} \leq 20 \mathrm{~K}$ is expected to have no significant influence on the martensite formation [4].

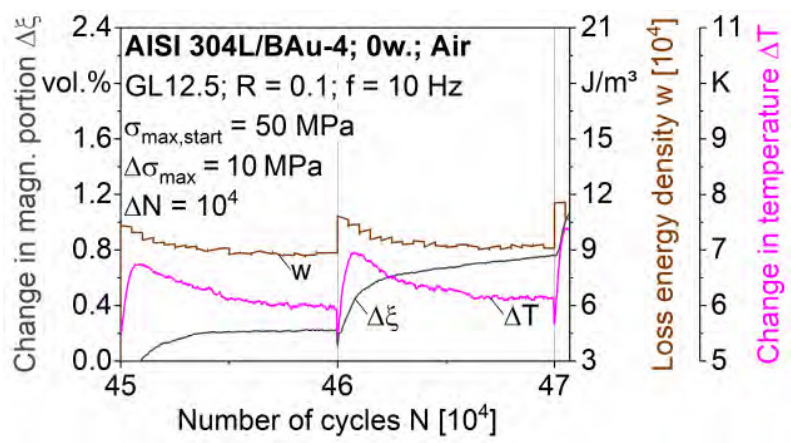

Fig. 9. Excerpt of the load increase test of a brazed specimen in the as-received condition.
Within LIT, a feritscope sensor was positioned at the base material close to the brazing seam to detect magnetic contents close to the surface, which indicate a deformation-induced formation of ferromagnetic martensite. Exemplarily, the change in magnetic portion $\Delta \zeta$ is presented for two stress levels of $\sigma_{\max }=500$ and $510 \mathrm{MPa}$, close to the failure of the specimen, Fig. 9. Therefore, a degressive trend of the change in magnetic portion $\Delta \zeta$ is detected for both stress levels with a maximum change of $\Delta \zeta=0.8 \mathrm{vol} \%$. The formation of martensite is expected to be the reason for a cyclic hardening effect, leading to a regressive trend of the loss energy density, which can be detected due to a regressive change in temperature. Thus, a combination of the electrical and temperature measurements is most suitable to characterise the cyclic deformation behaviour of brazed joints with ratcheting and cyclic hardening effects.

In the literature, a particular evaluation method of LIT was recommended for the characterisation of the fatigue behaviour of metastable austenitic materials [6]. It was shown that the high cycle fatigue strength corresponds well with the stress level, at which the deformationinduced formation of martensite and the temperature decrease due to the cyclic hardening occurs for the first time [6]. For brazed AISI 304L/BAu-4 joints of the current study, a temperature decrease in combination with a decreasing loss energy density $\mathrm{w}$ was first detected within the stress level of $\sigma_{\max }=380 \mathrm{MPa}$. For validation, constant amplitude tests with a limited number of cycles of $\mathrm{N}=2 \cdot 10^{6}$ were performed, Fig. 10 . The S-N curve is described in a double logarithmic scale acc. to Basquin with $\sigma_{\max }=\mathrm{S}_{\mathrm{D}} \cdot\left(\mathrm{N}_{\mathrm{D}} / \mathrm{N}_{\mathrm{f}}\right)^{1 / \mathrm{k}}$ with the gradient $\mathrm{k}$ [8]. The fatigue strength at $2 \cdot 10^{6}$ cycles of $397 \mathrm{MPa}$ and the gradient $\mathrm{k}=12.4$ are determined. Thus, the LIT combined with strain, temperature and electrical measurements are most suitable to estimate the fatigue strength of brazed AISI 304L/BAu-4 joints with a deviation of $4 \%$.

The triggered DIC system was applied within LIT of the brazed specimens to evaluate the total maximum strain $\varepsilon_{\text {max, }}$ as a function of the load cycles $N$. The engineering tangential strains in tensile direction $\varepsilon_{x x}$ are computed for line elements with lengths in the range of 0.5 to $12.5 \mathrm{~mm}$ (LE0.5 to LE12.5) to evaluate the influence of the measuring length on the cyclic deformation and ratcheting fatigue behaviour, Fig. 11.

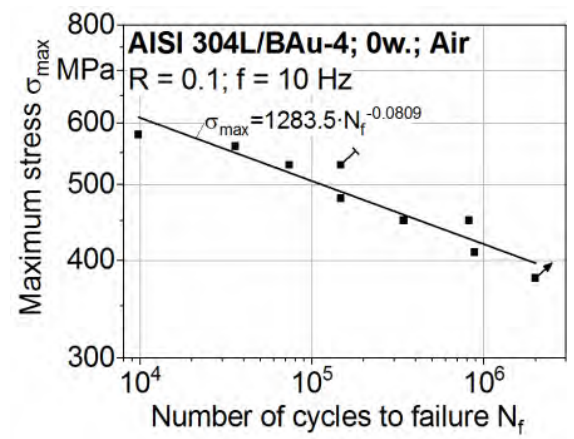

Fig. 10. Results of constant amplitude tests of brazed specimens in the as-received condition. 


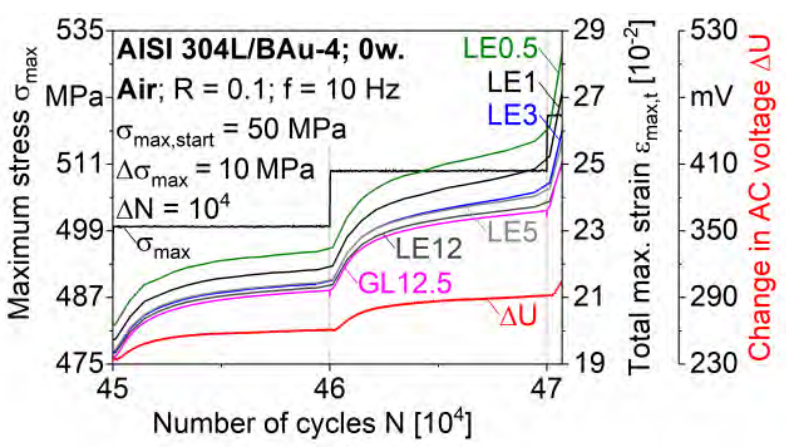

Fig. 11. Excerpt of the load increase test with a DIC analysis of a brazed specimen in the as-received condition.

When showing the total maximum strain $\varepsilon_{\text {max,t }}$ for the entire LIT for an as-received brazed specimen, a distinction between the values measured by the extensometer and computed by the DIC system is not possible. Therefore, an excerpt of the fatigue test results in the two stress levels before failure is illustrated, Fig. 11. Strain values, computed by the DIC system for a $12 \mathrm{~mm}$ line element (LE12), are in very good agreement with the results of the mechanical extensometer with a comparable gauge length (GL12.5). The comparison of the 0.5 and $5 \mathrm{~mm}$ line elements (LE0.5, LE5) for the two stress levels close to failure shows a maximum difference in strain values of $\Delta \varepsilon_{\text {max }, \mathrm{t}} \approx 3 \%$, Fig. 11 . For lower stresses, the difference in strain values is less pronounced and deviations $\Delta \varepsilon_{\text {max }, \mathrm{t}}<0.5 \%$ have to be considered for $\sigma_{\max }<$ $450 \mathrm{MPa}$.

The pre-corrosion leads to a significant influence on the fatigue behaviour with a reduction of the failure maximum stress down to $\sigma_{\max , \mathrm{f}(\mathrm{LIT})}=220 \mathrm{MPa}(42 \%)$, Fig. 12. The forces are related to the gross cross-section of the brazed joints. For $\sigma_{\max }=180 \mathrm{MPa}$, a transition from an almost steady to an increasing slope can be determined for the total maximum strain $\varepsilon_{\text {max,t, }}$, computed for the various line elements. In accordance with the tensile test results, the influence of the gauge length on the strain values is more pronounced for notch-containing conditions than for the as-received specimens, when considering comparable strain levels. Here, a decrease of the DIC line element length from 5 to $0.5 \mathrm{~mm}$ leads to a difference of $\Delta \varepsilon_{\text {max, }} \approx 3 \%$ at $17 \cdot 10^{4}$ cycles, close to failure. Values of the $12.5 \mathrm{~mm}$ line element (LE12.5) are in good accordance with the results of the extensometer with the same gauge length (GL12.5).

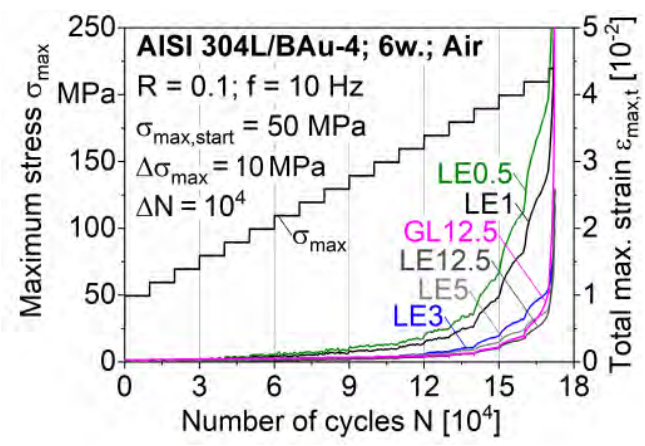

Fig. 12. LIT of a brazed specimen after pre-corrosion.

\subsection{Fractographic investigations}

Fracture surfaces of the brazed specimens were investigated using SEM in order to evaluate the fatigue and corrosion fatigue damage mechanisms. By using the BSE detector, the gold-based filler metal appears light grey in contrast to the dark grey stainless steel. On the topview of a specimen in the as-received condition, the fatigue fracture area at the top of the figure can be distinguished from the forced fracture area, which shows pronounced deformation characteristics, Fig. 12a. The fatigue crack initiated at the surface of the specimen in the area of a geometrical imperfection of the brazing seam and exposes a free solidified surface of the filler metal at the fracture surface, Fig. 12b. After initiation, the crack propagated close to the interface between the base material and the filler metal, detected by BSE as spotted light and dark grey areas, and in the base material, respectively, Fig. 12d. In contrast, the residual forced fracture occurred in the centre of the brazing seam, Fig. 12c.

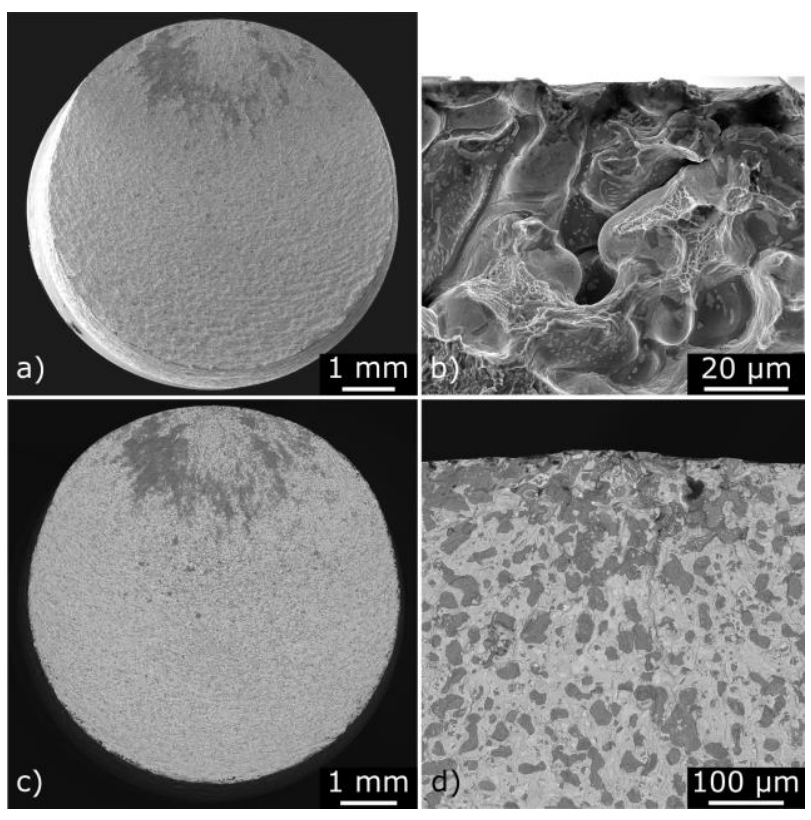

Fig. 13. Fracture surface of a brazed fatigue specimen in the as-received condition: Overview ( $a, c)$ and area of crack initiation (b, d); SEM with SE (a, b) and BSE (c, d).

Specimens, cyclically tested after 6 weeks of pre-corrosion, show a clearly visible corrosive attack, that progressed from the specimen's surface to the inner volume in the area of the brazing seam and significantly reduced the unaffected cross-section area, Fig. 14a. Within the circumferentially corroded region of the fracture surface, the filler metal can be distinguished from the two interfaces where the corrosive attack occurs locally. As the crack propagated alternatingly through one of the interfaces, the corroded base material below, as well as the corrosion products of the upper interface, can be identified, Fig. 14c.

Concerning the unaffected cross-section area, fracture characteristics comparable to the as-received specimens can be determined. Within the fatigue fracture area, which is positioned on the left side of Fig. 14b, the 
fatigue crack propagated preferentially through the base material, while the residual forced fracture occured in the centre of the brazing seam. In contrast to the crack initiation at one local imperfection close to the surface of the as-received specimens, a crack initiation zone within the circumferentially corroded interface leads to a minimum of six dark grey fatigue fracture areas that can be differentiated on the left side of the BSE image from the adjacent light grey residual forced fracture area in the centre of the brazing seam. A distinct transition from the forced fracture to the circumferentially corroded interface can be seen on the right side of the top-view.
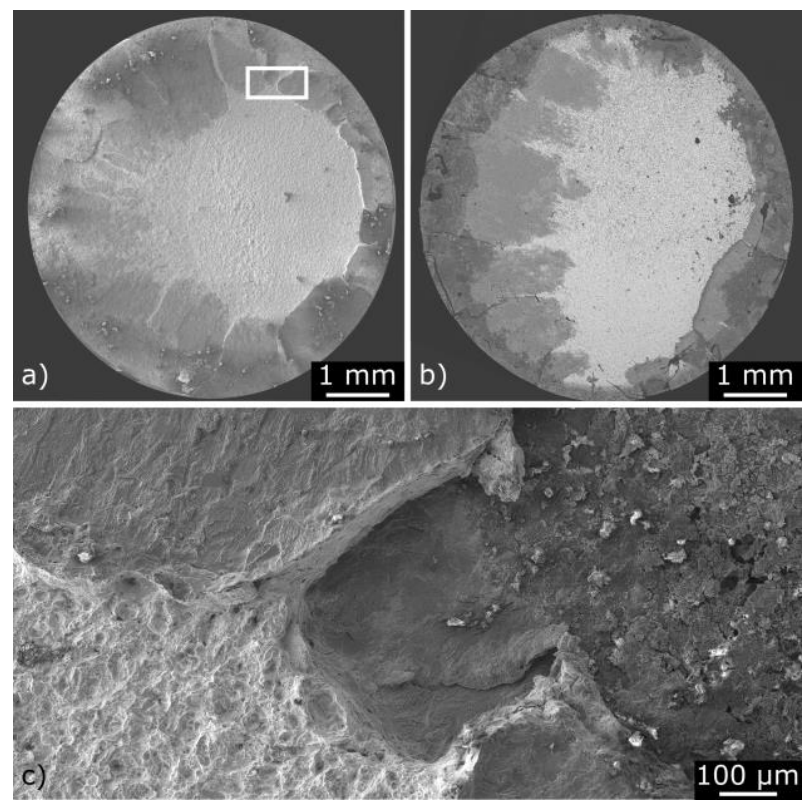

Fig. 14. Fracture surface of a pre-corroded brazed specimen after fatigue testing; SEM with SE (a, c) and BSE (b).

\section{Conclusions}

Pre-corrosions acc. to VDA 230-214 lead to a significant corrosive attack of brazed AISI 304L/BAu-4 joints, localised at the interface between the base material and the brazing seam. Compared to the as-received condition, the pre-corrosion leads to a reduction of the ultimate tensile strength $\sigma_{U T S}$ and the failure maximum stress $\sigma_{\max , \mathrm{f}(\mathrm{LIT})}$ down to $65 \%$ and to $42 \%$. In contrast, the pre-corrosion shows no influence on the tensile behaviour of the AISI 304L base material. In future studies, stress concentrations at the corrosion-dependent grooves of the brazed joints have to be evaluated.

LIT combined with strain, electrical, magnetic, and temperature measuring techniques have proven to be a most suitable approach to characterise the deformation and damage behaviour of brazed stainless steel joints. An evaluation method [6], developed for metastable austenitic materials, was successfully applied for the brazed specimens in order to estimate the fatigue strength with a deviation of $4 \%$. Further, the LIT enables a time-efficient assessment of the DIC technique for brazed joints. An optimised triggered image acquisition was applied to investigate local ratcheting fatigue effects at the brazing seam and the gauge length influence.
It was shown that the DIC technique is well appropriate to characterise the local deformation behaviour of brazed stainless steel joints within tensile and fatigue tests. Visualised local strain concentrations in the area of the brazing seam are more pronounced with increasing mechanical stresses and are significantly enhanced due to notch-containing surfaces. In general, strain values, computed by the DIC system, are in good agreement with the results of mechanical extensometers.

For brazed AISI $304 \mathrm{~L} / \mathrm{BAu}-4$ joints in the as-received condition, small differences in the strain values, computed for the gauge lengths of 0.5 and $5 \mathrm{~mm}$ with the DIC system, have to be considered. In tensile tests, the total strains at $\sigma_{\text {UTS }}$ differ with $\Delta \varepsilon_{\mathrm{t}} \approx 5 \%$ and the total maximum strains within LIT close to failure differ with $\Delta \varepsilon_{\text {max }, \mathrm{t}} \approx 3 \%$. There is no effect of the gauge length on the elastic modulus. For tensile and fatigue tests of pre-corroded brazed joints, a considerable influence of the gauge length on the strain values was determined due to splitting effects at the notches. Although the current study allows a fundamental comprehension of local strain concentrations based on $0.5 \mathrm{~mm}$ gauge lengths, local deformations within the $50 \mu \mathrm{m}$ brazing seam have to be evaluated in the future. Furthermore, the applicability of the DIC technique has to be evaluated for brazed joints with less pronounced deformation behaviours, such as AISI 304L/BNi-2 joints.

\section{References}

1. M. Koster, C. Kenel, C. Leinenbach, ICF13 (2013).

2. C. Leinenbach, M. Koster, H.J. Schindler, J Mater Eng Perform 21, 739 (2012).

3. D. Chen, S. Sun, J.M. Dulieu-Barton, Q. Li, W. Wang, Int J Fatigue 110, 172 (2018).

4. M. Smaga, F. Walther, D. Eifler, Materials Science and Engineering: A 483, 394 (2008).

5. T. Nebel, D. Eifler, Sadhana 28, 187 (2003).

6. C. Müller-Bollenhagen, Dissertation, University Siegen (2010).

7. I. Nikitin, M. Besel. Int. J. Fat. 30, 2044 (2008).

8. A. Schmiedt, M. Manka, T. Tillmann, F. Walther, Materialwiss Werkst, 10.1002/mawe201800007 (2018).

9. VDA Test Sheet 230-214, Verband der Automobilindustrie (2010).

10. F. Walther, Mater. Test. 56, 519 (2014).

11. A. Schmiedt, M. Manka, T. Tillmann, F. Walther, Weld World, 10.1007/s40194-018-0557-y (2018).

12. F. Hahnenberger, M. Smaga, D. Eifler, Int J Fatigue 69, 36 (2014).

\section{Acknowledgements}

The financial support of the German Research Foundation (DFG) is gratefully acknowledged (WA 1672/13, TI 343/96). The authors thank the Institute of Mechanics (IM) at TU Dortmund University for the provision of high resolution thermal camera. 